\title{
Hand Gesture Segmentation Method Based on YCbCr Color Space and K-Means Clustering
}

\author{
Zhang Qiu-yu, Lu Jun-chi, Zhang Mo-yi, Duan Hong-xiang and Lv Lu \\ School of Computer and Communication, Lanzhou University of Technology, \\ Lanzhou, 730050, China \\ zhangqylz@163.com,lujunchi9@163.com
}

\begin{abstract}
Aiming at the problems that current skin-color detection segmentation technologies have unsatisfied segmentation results under conditions of complex illumination or backgrounds, we present a new method based on $\mathrm{YCbCr}$ color space and $\mathrm{K}$-means clustering algorithm for segmentation hand gesture. Firstly, image in RGB color space is converted to $\mathrm{YCbCr}$ color space; and then $\mathrm{YCbCr}$ color space of image is divided into luminance $\mathrm{Y}$ and chrominance $\mathrm{Cb}$ and $\mathrm{Cr}$. Lastly, the binary image is achieved by clustering values of chrominance using k-means clustering algorithm, and hand gesture segmentation is completed by conducting morphological process of binary image obtained. The experimental results illustrate that the proposed method can segment hand gestures from complex backgrounds and obtain segmentation results. The phenomena of similar skin color interference and skin color overlapping are solved with this method effectively. In addition, it is robust to illumination condition.
\end{abstract}

Keyword: Hand gesture segmentation, $\mathrm{YCbCr}$ color space, K-means clustering, Morphological processing

\section{Introduction}

In the hand recognition system, hand gesture segmentation is basic and key to step of the whole hand gesture recognition. Hand gesture segmentation results will influence follow-up hand recognition procedure directly [1]. Therefore, the stage of hand gesture segmentation needs to improve the segmentation accuracy, shorten segmentation time, reduce the effect of illumination conditions on hand gesture segmentation process and segment hand gesture from complex backgrounds or different illumination conditions. Hand gesture segmentation technology has a crucial place in the field of hand gesture recognition $[2,3]$.

In recent years, many researchers have used color spaces to segment hand gesture and obtained hand gesture segmentation images by setting skin color threshold [2-4]. Because the skin color of hand is within a constant threshold range, using hand skin threshold method can segment hand gesture from backgrounds simply and fast. However, only using skin-color threshold method will also cause some problems, such as the result of hand gesture segmentation affected by illumination conditions easily, too many noise effects, complex backgrounds or similar skin color interference. It is hard to segment hand gesture from backgrounds in such conditions. Therefore, many researchers utilized methods that combined color spaces and image segmentation methods to segment hand gesture. Ramakrishnan, et al., [3] proposed to utilize Cybergloves to get hand gesture information by collecting hand shapes joints and mobile hand details, and then established hand gesture 3D models. They applied Principal Component Analysis method to reduce dimensions and segment hand gesture. Using Cybergloves can avoid effects of hand gesture backgrounds and experiment illumination. However, this kind of sensor used in the hand segmentation procedure cannot satisfy the demand of nature human-computer 
interaction. Wang, et al., [5] used RGB color space to segment hand gesture, combining clustering character of $\mathrm{YCbCr}$ color space, and achieved color extraction. After extracting color image, they continued to detect edge and fill the binary holes with morphology processing. Fang, et al., [6] applied classic $\mathrm{YCbCr}$ color space. They equalized chrominance $\mathrm{Cb}$ and $\mathrm{Cr}$ from $\mathrm{YCbCr}$ color space and obtained binary hand segmentation result by regarding the value of chrominance $\mathrm{CbCr}$ as the parameters of elliptic equation model. Zhang, et al., [7] utilized skin detection in HSV color space and circular gradient edge detection technology to segment hand gesture. Their proposed method transformed RGB color space to HSV color space and set color threshold in the HSV color space. After color threshold filtering, they finished the hand skin detection and then selected seed points in the hand images region by using circular gradient edge detection technology. They detected skin edge with seed point center and filled the binary hand segmentation holes. Tan, et al., [8] proposed a method that combined GVF Snake and skin model to extract hand gesture contour. Sgouropoulos, et al., [9] applied face detection technology through skin color detection and filtered out human face firstly. They segmented hand gesture form human hand region in $\mathrm{YCbCr}$ color space. All these methods mentioned above can segment hand gesture from image backgrounds. However, most of these methods finish segmenting hand gesture in condition of white or unlike skin color backgrounds.

Aiming at disadvantages mentioned above, this paper proposes a hand segmentation method based on $\mathrm{YCbCr}$ color space and $\mathrm{K}$-means clustering algorithm. Firstly, the proposed method transforms traditional RGB color space model to HSV color space and $\mathrm{YCbCr}$ color that are widely used. By comparing different color spaces features, this paper selects proper color space for hand gesture segmentation. Then obtain chrominance $\mathrm{Cb}$ and $\mathrm{Cr}$ from selected $\mathrm{YCbCr}$ color space and cluster chrominance information with K-means clustering algorithm. Lastly, process the image after clustering with morphological method and finish hand gesture segmentation. The experiment shows that the proposed method can segment hand gesture from complex backgrounds and different illumination conditions effectively. It also gets hand segmentation binary images. By observing experiment results, it is proved that the proposed method has practical value.

\section{Color Spaces}

For computer images, there form different color spaces depending on different color expression ways $[2,4,10]$. Hand skin has different color ranges in different color spaces. So selecting appropriate color space is the key stage for hand segmentation procedure. For now, most of researchers use RGB, HSV and YCbCr color spaces in the field of skin color detection of hand gesture.

\subsection{RGB Color Space}

RGB color space is a kind of mixed color space, describing color space through red green blue primary colors, and can represent most of colors. However, RGB color space is not used in most experiments. Because it is difficult to digitize the details and RGB color space mixes hue, luminance and saturation together. Each color channel is highly correlated and dependent, which means current methods can separate them hardly. In 3D space, RGB color space can be represented as a cube shown in Figure 1. 


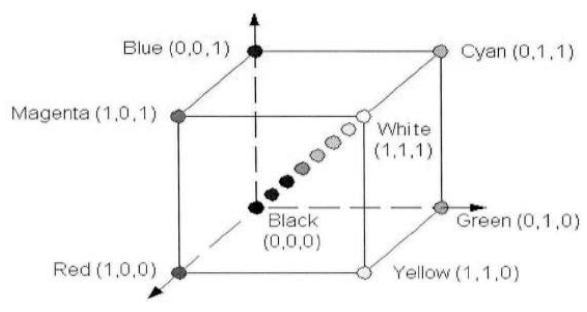

Figure 1. RGB Color Space

\subsection{HSV Color Space}

HSV color space is a kind of intensity/saturation/hue color space. HSV color space utilizes hue, saturation and value to express colors. Hue $H\left[0^{\circ}, 360^{\circ}\right]$ is described by angle. Saturation $S$ (color purity) means the distance to $V$ axis. Value $V$ is luminance. Convert RGB color space to HSV color space. Formulas are as follows.

Firstly, RGB color space is normalized, which makes $R G B \in[0,1] . \max =\max (R, G$, $B), \min =\min (R, G, B)$.

$$
\begin{aligned}
& \mathrm{H}=\left\{\begin{array}{l}
\text { undefined when } S=0 \\
\bmod \left\{\frac{G-B}{\max -\min } \times 60,360\right\}, \text { when } R=\max \\
2+\bmod \left\{\frac{G-B}{\max -\min } \times 60,360\right\}, \text { when } G=\max \\
4+\bmod \left\{\frac{G-B}{\max -\min } \times 60,360\right\}, \text { when } B=\max
\end{array}\right. \\
& \mathrm{S}=\left\{\begin{array}{l}
0, \text { when } V=0 \\
\frac{\max -\min }{\max }, \text { when } V \neq 0
\end{array}\right. \\
& \mathrm{V}=\max (R, G, B)
\end{aligned}
$$

HSV color space converted from RGB color space can be represented as a cone. $H$ is the angle between bottom radius and $0^{\circ}$ line, $S$ is the length of cone radius, $V$ is the height. As is shown in Figure 2.

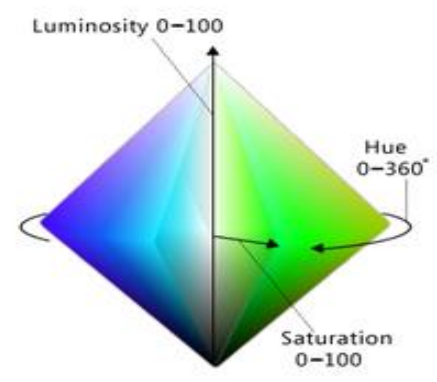

Figure 2. HSV Color Space

\subsection{YCbCr Color Space}

$\mathrm{YCbCr}$ color space is a kind of linear luminance/chromaticity color space. Moreover, it is one of the YUV color space family. In $\mathrm{YCbCr}$ color space, $\mathrm{Y}$ is luminance. $\mathrm{Cb}$ and $\mathrm{Cr}$ are chromaticity of blue and red colors. $\mathrm{Cb}$ and $\mathrm{Cr}$ are two-dimensional independent. Convert RGB color space to $\mathrm{YCbCr}$ color space. Converting formula is 


$$
\left[\begin{array}{c}
\mathrm{Y} \\
C b \\
C r
\end{array}\right]=\left[\begin{array}{ccc}
0.2990 & 0.5870 & 0.1440 \\
-0.1687 & -0.3313 & 0.5000 \\
0.5000 & -0.4187 & -0.0813
\end{array}\right]\left[\begin{array}{l}
R \\
G \\
B
\end{array}\right]+\left[\begin{array}{c}
0 \\
128 \\
128
\end{array}\right]
$$

\subsection{Color Spaces Analysis and Selection}

In the procedure of hand gesture segmentation, luminance value will influence experiment results of hand gesture segmentation. Therefore, the selected color space should avoid the effect of luminance. In addition, the selected color is supposed to have good clustering feature.

RGB color space is the color space model that mixes three kinds of colors information. Clustering range of the same skin varies a lot in RGB color space. In the RGB color space, skin range and luminance are highly correlated.

Comparing these situations, HSV color space and $\mathrm{YCbCr}$ color space have better clustering features than RGB color space. Comparing formulas from (1) to (4), it can be concluded that HSV color space is continuous space and $\mathrm{YCbCr}$ color space is discrete space. Luminance $\mathrm{Y}$ and color chromaticity $\mathrm{CbCr}$ are obtained through linear transformation of RGB primary colors. Using discrete $\mathrm{YCbCr}$ color space can get better clustering results and computational efficiency. According to the comparison of three kinds of color spaces, This paper chooses $\mathrm{YCbCr}$ color space for hand gesture segmentation.

\section{Hand Gesture Segmentation Method Based on YCbCr Color Space and K-means Clustering}

\subsection{Hand Segmentation Processing Procedure}

Firstly, convert RGB color space images to $\mathrm{YCbCr}$ color space images. Then regard chromaticity value as the initial database of K-means clustering algorithm and carry on binary process. Lastly, get rid of binary image holes with morphological process and finish hand gesture images segmentation.

Hand segmentation procedure chart shown in Figure 3.

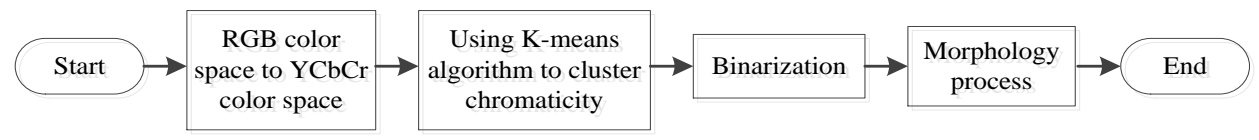

Figure 3. Hand Segmentation Procedure

\subsection{Color Space Means Clustering}

K-means algorithm [11], a sort of classic clustering algorithm, was proposed by MacQueen J in 1967, which belongs to distance clustering algorithm. Because K-means algorithm has features of simplicity and efficiency, it is widely applied in the procedure of massive images clustering [11].

The basic idea of K-means algorithm: K-means algorithm is a kind of hard clustering algorithm, which means that it divides $\mathrm{n}$ data samples of $n$-dimensional Euclidean space into $K$ classes. Firstly, determine accurate clustering number $K$ and select $K$ objects randomly as center points. Then put the point to the nearest class by judging the distance between data point and center point and then recalculate the average of the new clustering center in each class. Finally, iterate until the objective function converges [12].

$\mathrm{K}$-means algorithm needs to allocate and adjust all the data points in each procedure of iteration, calculate the clustering center and continue next iteration procedure. If there is 
no change of location in one procedure, then there is no corresponding change in the clustering center. That means clustering function is convergence and clustering algorithm ends $[13,14]$.

In the K-means algorithm, define the set as follows.

$$
\mathrm{X}=\left(x_{1}, x_{2}, \cdots, x_{n}\right)
$$

where $X$ is the initial data object sets.

Euclidean distance of any two of object data in the object database is

$$
\mathrm{d}_{\mathrm{ij}}=\sqrt{\sum_{k=1}^{n}\left(x_{i k}-x_{j k}\right)^{2}}
$$

where $x_{\mathrm{i}}=\left(x_{\mathrm{i} 1}, x_{\mathrm{i} 2}, \ldots, x_{\mathrm{in}}\right)$ and $x_{\mathrm{j}}=\left(x_{\mathrm{j} 1}, x_{\mathrm{j} 2}, \ldots, x_{\mathrm{jn}}\right)$ are two $n$-dimensional object data. $x_{\mathrm{i} 1}$, $x_{\mathrm{i} 2}, \ldots, x_{\mathrm{in}}$ and $x_{\mathrm{j} 1}, \mathrm{x}_{\mathrm{j} 2}, \ldots, x_{\mathrm{jn}}$ are similarities of samples of $x_{\mathrm{i}}$ and $x_{\mathrm{j}}$. Judge samples similarities by calculating the distance $d_{\mathrm{ij}}$ between $x_{\mathrm{i}}$ and $x_{\mathrm{j}}$. The smaller the distance $d_{\mathrm{ij}}$ is, the greater the degree of similarity is. In this experiment, $x_{\mathrm{i}}$ and $x_{\mathrm{j}}$ are the chromaticity information of each point.

The same class center point:

$$
\mathrm{C}_{j}=\frac{1}{n_{j}} \sum_{x \in w_{j}} x
$$

where $n_{\mathrm{j}}$ is the number of the same class, $w_{\mathrm{j}}$ is the K clustering category.

The objective function is defined as:

$$
\mathbf{J}=\sum_{i=1}^{K} \sum_{j=1}^{n_{j}} d\left(x_{i}, x_{j}\right)
$$

where $d_{\mathrm{ij}}\left(x_{\mathrm{i}}, x_{\mathrm{j}}\right)$ is the shortest distance between $x_{\mathrm{i}}$ and $x_{\mathrm{j}}$. Namely, the distance is the Euclidean distance between $x_{\mathrm{i}}$ and $x_{\mathrm{j}}$. Objective function $J$ is the clustering result after calculating.

K-means algorithm procedure:

Input parameters: $K$, the number of clustering. Object data sets $X=\left(x_{1}, x_{2}, \ldots, x_{\mathrm{n}}\right)$;

Output parameters: $K$ classes $C_{\mathrm{j}}, j=1,2, \ldots, K$;

Step 1: According to the value $K$ of clustering set, select $K$ data from data sets randomly as initial clustering center of data sets;

Step 2: Calculate the Euclidean distance between $K$ initial clustering centers and data of samples set $X=\left(x_{1}, x_{2}, \ldots, x_{n}\right)$. Judging by the shortest distance principle, the data samples are reclassified to the nearest class;

Step 3: Calculate the average of samples of each class as new $K$ clustering centers;

Step4: Repeat Step2 and Step3 until clustering centers are not changing. In addition, objective function $J$ does not change;

Step 5: End clustering procedure and obtain $K$ clustering results.

$\mathrm{K}$-means clustering algorithm flow chart is shown in Figure 4. 


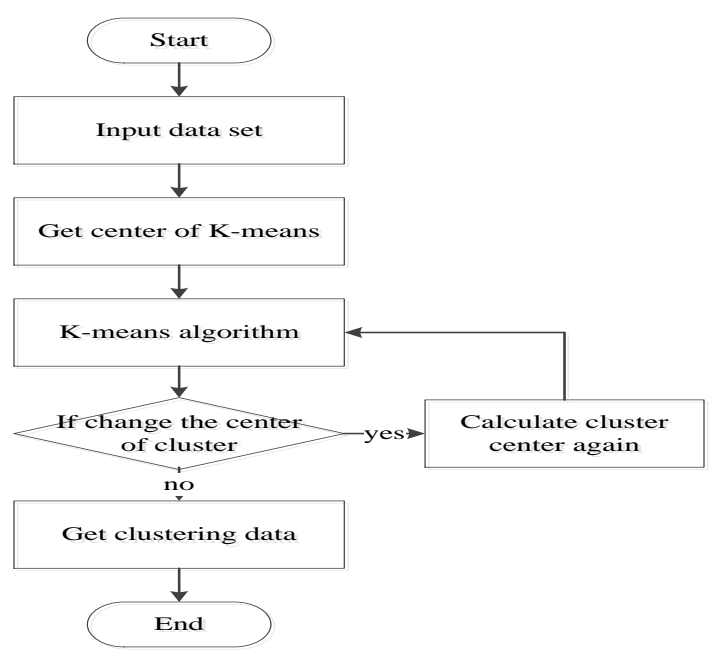

Figure 4. K-means Clustering Algorithm

K-means algorithm is the clustering algorithm based on partition. This algorithm clusters through constant iterative process. End iteration procedure when the algorithm converges to an end condition. Then output clustering results. For dealing with massive database of images, this algorithm is scalable and efficient.

\subsection{K-means Clustering of Chromaticity Value}

Based on features of K-means mentioned above, this paper utilizes K-means clustering algorithm to cluster chromaticity information. $\mathrm{YCbCr}$ color space transformed from RGB color space is not suitable for clustering algorithm. Because the luminance $Y$ of $\mathrm{YCbCr}$ color space images has great effect on the results of hand gesture segmentation. Therefore, this paper separates luminance $\mathrm{Y}$ from $\mathrm{YCbCr}$ images to avoid effects on hand gesture segmentation results. With the chromaticity remained, the proposed method not only reduces the effect on hand gesture segmentation result but also keeps chromaticity information of $\mathrm{YCbCr}$ images and human hand skin feature especially. The purpose of hand gesture segmentation is to segment hand gesture from backgrounds. Therefore, this paper divides hand images into two classes. One class is foreground hand, and another class is background image. According to ideas above, parameter of K-means algorithm is set for 2. Namely, the value $K$ is 2 . In this way, the purpose that distinguishes foreground and background will be done. Select chromaticity separated from YCbCr color space as the initial data samples of K-means clustering algorithm. Divide chromaticity image into two categories through $\mathrm{K}$-means clustering algorithm that parameter $K$ equals 2 . In addition, obtain foreground hand gesture and background.

Firstly, get chromaticity mean clustering result of original hand gesture image through parameter $K$ and finish hand gesture image clustering manipulation. Then carry on binarization process after clustering and get initial results of hand gesture segmentation. The effect on segmentation results of luminance is avoided in this way.

\subsection{Morphology Process}

Binary image morphology is a most basic morphology theory. Binary image means that gray image can only have two possible values [15]. Its basic idea is to use a certain form of structural elements to measure and extract the corresponding shape of image so that it can achieve the purpose of processing and analyzing image [16]. Math morphology includes dilation, erosion, opening and closing operation. Dilation and erosion operation, as the base of math morphology, can highlight useful information of images [17, 18]. 
Set $A \in E^{\mathrm{n}}$. Structural elements $B \in E^{\mathrm{n}}$ or points set of subspace. Two vectors $a$ and $b$ are elements of $A$ and $B$. The definition of dilation and erosion based on Minkowski sum and difference is:

$$
\begin{aligned}
& \mathrm{A} \oplus \mathrm{B}=\left\{\mathrm{z} \in \mathrm{E}^{\mathrm{n}}: \mathrm{z}=\mathrm{a}+\mathrm{b}, \mathrm{a} \in \mathrm{A}, \mathrm{b} \in \mathrm{B}\right\}=\bigcup_{b \in B}(A)_{b} \\
& A \Theta B=\left\{z \in E^{n}: z=a+b \in A, \forall b \in B\right\}=\bigcap_{b \in B}(A)_{-b}
\end{aligned}
$$

where $(A)_{\mathrm{b}}$ is the pan conversion of vector $b$ to set $A$.

$$
(A)_{b}=\left\{z \in E^{n}, z=a+b, a \in A\right\}
$$

Apply dilation and erosion definition of Minkowski into binary images. The new definitions of dilation and erosion are below:

$$
\begin{gathered}
\mathrm{A} \oplus \mathrm{B}=\left\{\mathrm{z} \in \mathrm{E}^{\mathrm{n}}: \mathrm{z}=\mathrm{a}+\mathrm{b}, \mathrm{a} \in \mathrm{A}, \mathrm{b} \in \mathrm{B}\right\}=\bigcup_{b \in B}(A)_{b}=\{\mathrm{x}[(\hat{\mathrm{B}}) \mathrm{x} \cap \mathrm{A}] \neq \phi\} \\
A \Theta B=\left\{z \in E^{n}: z=a+b \in A, \forall b \in B\right\}=\bigcap_{b \in B}(A)_{-b}\{\mathrm{x} \mid[(\mathrm{B}) \mathrm{x} \cap \mathrm{A}]=\phi\}
\end{gathered}
$$

Suppose $A$ is an image, $b$ is structural element, $\hat{B}$ is the mapping of $B$.

$$
\hat{\mathrm{B}}=\{x \mid x=-b, b \in B\}
$$

Binary erosion operation has effects on eliminating the boundary points of the object in math morphology operation. Erosion operation can eliminate the result that is smaller than the structural element. In this way, selecting structural elements with different sizes can eliminate objects with different sizes in the original images. If there is tiny connectivity between two objects, separate two objects with erosion operation when the structural element is big enough. It illustrates that erosion operation has the effect on eliminating noise. Erosion operation can also fill holes inside images when structural elements do not include origin.

Binary dilation operation is different from filtering operation for external images. If structural element is a round, dilation operation can be used to fill images whose holes are bigger than structural element and the small portion of image edge can be processed too. Dilation operation can also connect two objects with relatively close distance [19, 20].

Under natural conditions, the illumination environment of hand gesture experiment is uneven and intensity of illumination is uncertain. These conditions will bring optical noise to hand gesture images, which has great effect on binary images of hand gesture segmentation. In addition, surface of hand skin and experiment background will be reflective. The phenomenon of uneven illumination will influence the experiment results of hand gesture segmentation. using median filtering methods or illumination compensation to process original RGB hand gesture images can reduce effects of these factors on experiments. Nevertheless, using median filtering methods or illumination compensation method to process RGB color images will bring other problems. The processing images procedure of these two kinds of methods will cause additional time consuming on segmentation experiment, which makes the real-time unavailable. Therefore, use morphology method to process binary images so that it can achieve the purpose of handling images interference and reducing time consuming.

Based on the effects and features of dilation and erosion operation, the binary image obtained after K-means clustering algorithm is made as the object of morphology dilation and erosion operation. And continue to process binary image. The whole segmentation procedure is not doing any removing noise processing on the original hand gesture image of RGB color space. Therefore binary hand gesture image obtained after K-means clustering algorithm has many interference points, such as many holes in the binary image, binary hand gesture jagged edge. To get rid of these interference effects on the binary hand gesture image, process binary hand images with morphology dilation and erosion methods. Moreover, process holes, jagged edge and finger adhesions of binary hand gesture image with structural elements of morphology algorithm. Reducing these 
effects on experiment results, good binary hand gesture segmentation images can be got and binary hand gesture can be applied to hand recognition perfectly.

\section{Experiment Results and Analysis}

Experimental hardware platform: Inter Core i3, 2450M, 2G, 2.27GHz. Experiment environment: Windows 7 system and OpenCV. Data base of experiment one and two are single hand gesture images of different backgrounds and illumination conditions. Image resolution: $320 \times 240$. Data base of experiment three and four are from Jochen Triesch Static Hand Posture Database II of http://www.idiap.ch/resource/gestures. Image resolution of experiment three: $128 \times 128$. Image resolution of experiment four: $384 \times 288$.

-Experiment One: Segment hand gesture from different backgrounds under uniform illumination condition.

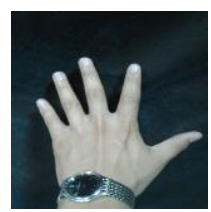

(a) dark green

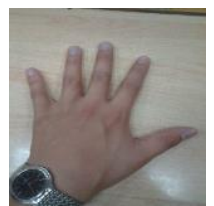

(b) similar skin

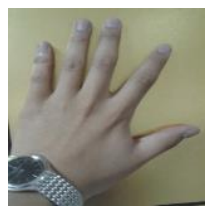

(c) yellow

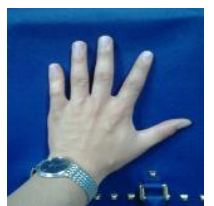

(d) blue

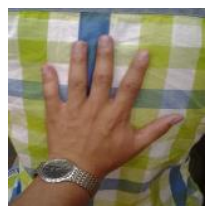

(e) motley

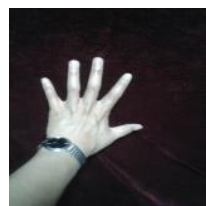

(f) dark red

Figure 5. Complex Backgrounds used in Experiment

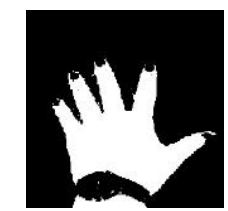

(a) Figure 5(a)

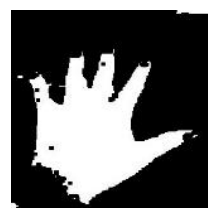

(b) Figure 5(b)

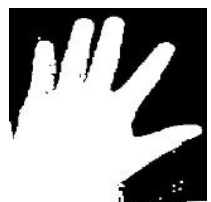

(c) Figure 5(c)

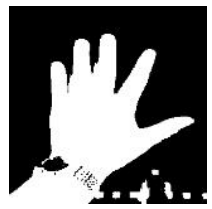

(d) Figure 5(d)

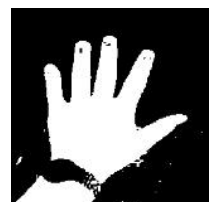

(e) Figure 5(e)

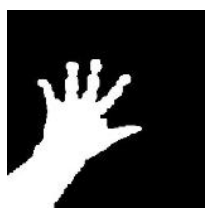

(f) Figure 5(f)

Figure 6. Segmentation Results under Complex Backgrounds Condition

Table 1. Hand Gesture Segmentation Time with Different Backgrounds

\begin{tabular}{ccccccc}
\hline Background & Figure 5(a) & Figure 5(b) & Figure 5(c) & Figure 5(d) & Figure 5(e) & Figure 5(f) \\
\hline Time $(m s)$ & 698 & 648 & 719 & 716 & 1268 & 650 \\
\hline
\end{tabular}

-Experiment Two: Segment hand gesture from white background under different illumination conditions

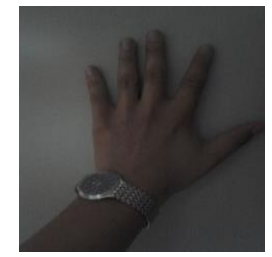

(a) dark condition 1

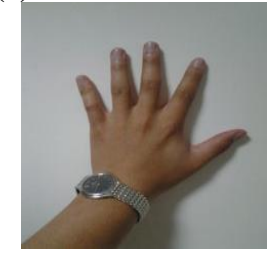

(e) normal condition1

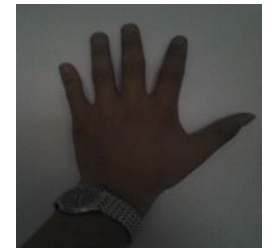

(b) dark condition 2

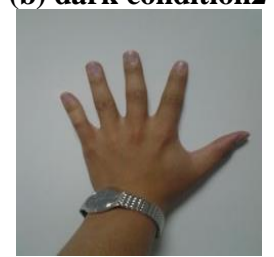

(f) normal condition2

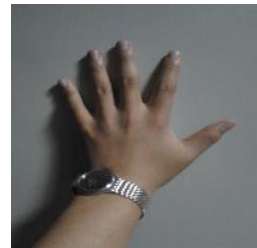

(c) dark conditon 3

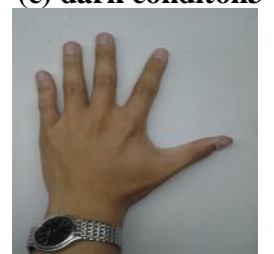

(g) glare condition 1

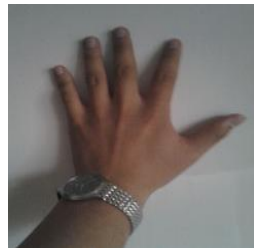

(d) dark condition 4

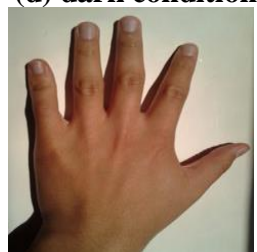

(h) glare conditon2

Figure 7. Different Illumination used in Experiment 


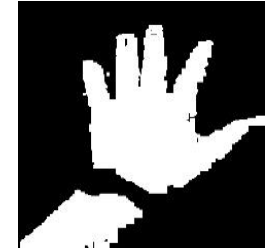

(a) Figure 7(a)

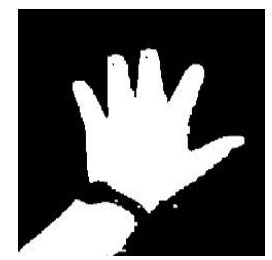

(e) Figure 7(e)

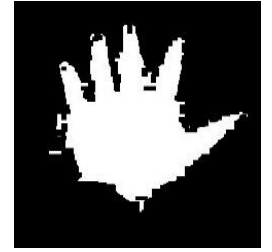

(b) Figure 7(b)

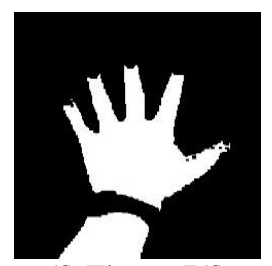

(f) Figure 7(f)

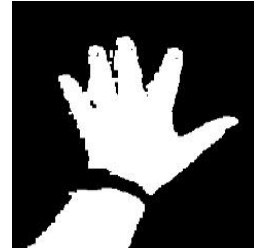

(c) Figure 7(c)

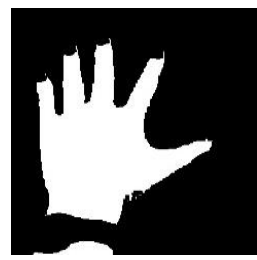

(g) Figure 7(g)

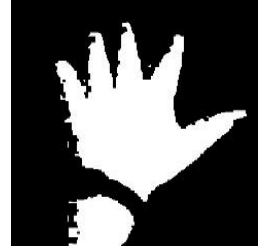

(d) Figure 7(d)

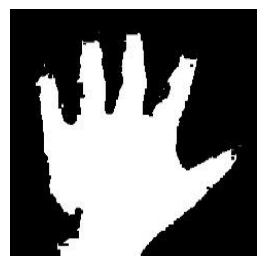

(h) Figure 7(h)

Figure 8. Segmentation Results under Different Illumination Conditions

Table 2. Hand Gesture Segmentation Time with Different Illumination

\begin{tabular}{ccccccccc}
\hline \multirow{2}{*}{ Illumination } & Figure & Figure & Figure & Figure & Figure & Figure & Figure & Figure \\
& $7(\mathrm{a})$ & $7(\mathrm{~b})$ & $7(\mathrm{c})$ & $7(\mathrm{~d})$ & $7(\mathrm{e})$ & $7(\mathrm{f})$ & $7(\mathrm{~g})$ & $7(\mathrm{~h})$ \\
\hline \multirow{2}{*}{ Time $(m s)$} & 817 & 689 & 753 & 735 & 709 & 689 & 773 & 800 \\
\hline
\end{tabular}

-Experiment Three: Hand gesture with different backgrounds 1(Jochen Triesch Static Hand Posture Database II)

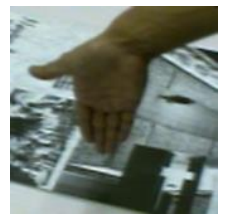

(a)

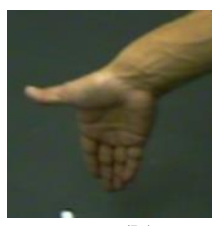

(b)

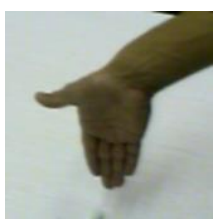

(c)

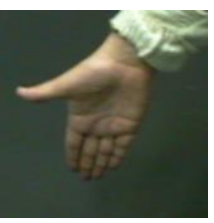

(d)

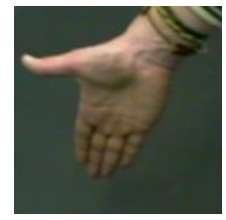

(e)

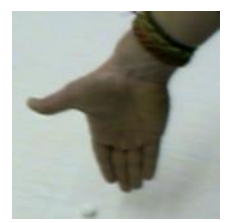

(f)

Figure 9. Hand Gesture 1 with Different Backgrounds in Hand Data Sets

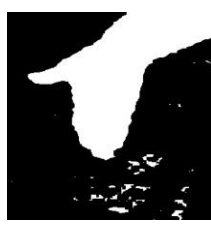

(a) Figure 9(a)

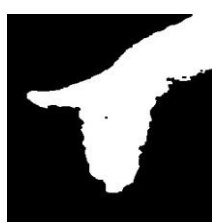

(b) Figure 9 (b)

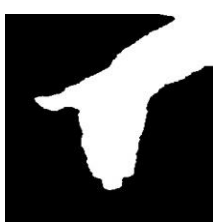

(c) Figure 9(c)

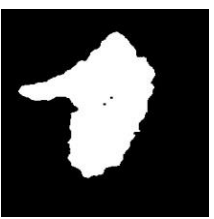

(d) Figure 9(d)

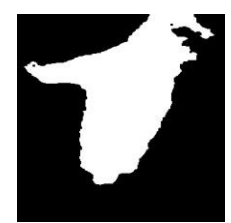

(e) Figure 9(e)

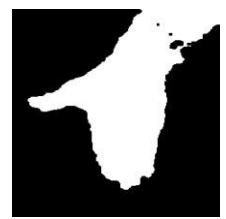

(f) Figure 9(f)

Figure 10. Segmentation Results 1 of Hand Data Sets

Table 3. Segmentation Time of Hand Data Sets 1

\begin{tabular}{ccccccc}
\hline Background & Figure 9(a) & Figure 9(b) & Figure 9(c) & Figure 9(d) & Figure 5(e) & Figure 9(f) \\
\hline Time $(m s)$ & 191 & 227 & 203 & 206 & 175 & 201 \\
\hline
\end{tabular}

- Experiment Four: Hand gesture with different backgrounds 2(Jochen Triesch Static Hand Posture Database II) 


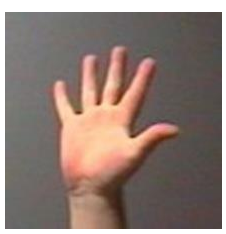

(a)

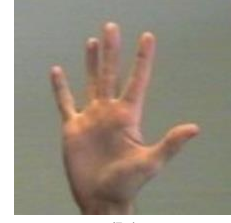

(b)

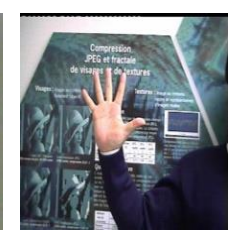

(c)

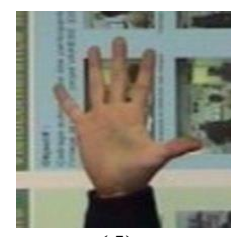

(d)

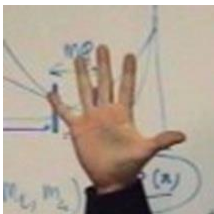

(e)

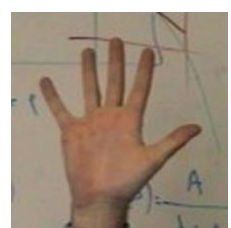

(f)

Figure 11. Hand Gesture 2 with Different Backgrounds in Hand Data Sets
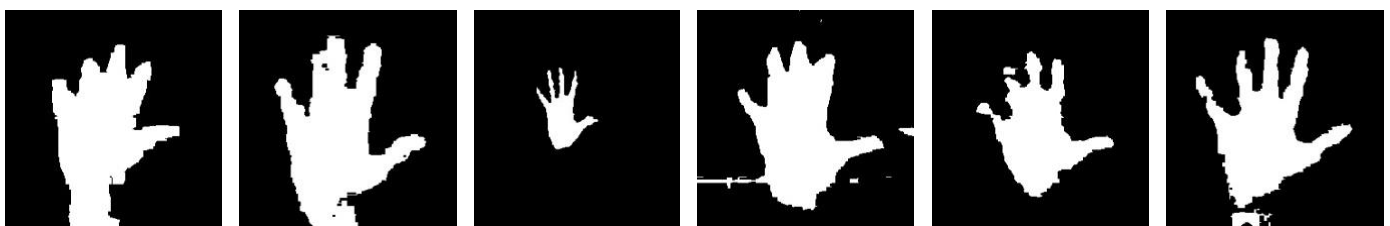

(a) Figure 11(a) (b) Figure 11(b) (c) Figure 11(c) (d) Figure 11(d) (e) Figure 11(e) (f) Figure 11(f)

Figure 12. Segmentation Results 2 of Hand Data Sets

Table 4. Segmentation Time of Hand Data Sets 2

\begin{tabular}{ccccccc}
\hline Background & Figure 11(a) & Figure 11(b) & Figure 11(c) & Figure 11(d) & Figure 11(e) & Figure 11(f) \\
\hline Time $(m s)$ & 668 & 923 & 831 & 875 & 813 & 679
\end{tabular}

In Experiment One, backgrounds of Figure 5 are different experiment backgrounds used in this paper. Use laboratory lights as experiment light source. Segment hand gesture under the condition mentioned above. It can be concluded from Figure 6 that applying the proposed method can segment hand gesture from complex backgrounds. Moreover, segmentation results are satisfactory. The consuming average time under complex backgrounds conditions is $783 \mathrm{~ms}$.

In Experiment Two, experiment images in Figure 7 have same background but different illumination conditions. White experiment wall is as experiment background and segment hand gesture with illumination darkening gradually. From segmentation results in Figure 8, this paper can segment hand gesture from backgrounds with different illumination conditions. After observing the experiment results, It can be concluded that the proposed method is robust to various illumination conditions. The consuming average time under different illumination conditions is $745 \mathrm{~ms}$.

In Experiment Three, hand gesture images in Figure 9 are from Jochen Triesch Static Hand Posture Database II. From segmentation results in Figure 10, a conclusion can be made that the proposed method can segment hand gesture from backgrounds in Jochen Triesch Static Hand Posture Database II. The consuming average time of segmenting hand gesture is $200 \mathrm{~ms}$.

In Experiment Four, hand gesture images " 5 " in Figure 11 are from Jochen Triesch Static Hand Posture Database II. From the hand segmentation results in Figure 12, a conclusion can be made that the proposed method can segment hand gesture from backgrounds in Jochen Triesch Static Hand Posture Database II. The consuming average time of segmenting hand gesture is $798 \mathrm{~ms}$.

\section{Conclusions}

This paper proposes a hand gesture method based on $\mathrm{YCbCr}$ color space and $\mathrm{K}$-means clustering algorithm. The proposed method can segment hand gesture from complex backgrounds and different illumination conditions. This method uses chromaticity of $\mathrm{YCbCr}$ color space, avoiding the effect on hand gesture segmentation, and uses k-means clustering algorithm to cluster chromaticity. Finally, use morphology method to process segmentation results and finish hand gesture segmentation. Experiment shows that this 
method has good segmentation results and robustness to complex backgrounds and illumination. Moreover, it has real-time.

How to segment hand gesture under complex situations and improve the real-time of images access will be the importance of continuous research.

\section{Acknowledgments}

This work is supported by the National Natural Science Foundation of China (No. 61363078), the Natural Science Foundation of Gansu Province of China (No. 1212RJZA006, No. 1310RJYA004). The authors would like to thank the anonymous reviewers for their helpful comments and suggestions.

\section{References}

[1] H. Li and M. Greenspan, "Model-based segmentation and recognition of dynamic gestures in continuous video streams", Pattern Recognition, vol. 44, no. 8, (2011), pp. 1614-1628.

[2] H. Duan and Y. Luo, "A Method of Gesture Segmentation Based on Skin Color and Background Difference Method", Proceedings of the 2nd International Conference on Computer Science and Electronics Engineering, (2013) March 22-23, Hangzhou, China.

[3] A. S. Ramakrishnan and M. Neff, "Segmentation of hand gestures using motion capture data", Proceedings of the 2013 International Conference on Autonomous Agents and Multi-agent Systems, (2013) May 6-10, Saint Paul, USA.

[4] C. L. Hwang, K. D. Lu and Y. T. Pan, "Segmentation of Different Skin Colors with Different Lighting Conditions by Combining Graph Cuts Algorithm with Probability Neural Network Classification, and its Application", Neural processing letters, vol. 37, no. 1, (2013), pp. 89-109.

[5] X. J. Wang, G. Z. Bai and Y. M. Yang, "Hand Gesture Recognition Based on BP Neural Network in Complex Background", Computer Applications and Software(in Chinese), vol. 30, no. 3, (2013), pp. 247-249.

[6] K. Fang, Y. N. Ou and J. W. Mo, "Gesture Recognition and Similation Based on Elliptical Model”, The computer simulation (in Chinese), vol. 28, no. 3, (2011), pp. 267-270.

[7] G. J. Zhang, D. W. Zuo, X. F. Li and C. H. Shi, "Design and Development of a New Gesture Segmentation Algorithm Based on Circular Gradient”, Machine Design and Manufacturing Engineering (in Chinese), vol. 42, no. 9, (2013), pp. 1-6.

[8] W. J. Tan, J. Z. Yang, X. M. Song and D. Z. Zhao, "Fusion Extraction Method of Gesture Contour Based on GVF Snake and Skin Color Model", Journal of Chinese Computer Systems (in Chinese), vol. 34, no. 6, (2013), pp. 1405-1408.

[9] K. Sgouropoulos, E. Stergiopoulou and N. A. Papamarkos, "A Dynamic Gesture and Posture Recognition System”, Journal of Intelligent \& Robotic Systems, vol. 76, no. 2, (2013), pp. 283-296.

[10] W. J. Tan, "Research on Algorithm and Model of Hand Gestures Recognition Based on Computer Vision", Northeastern University, Liaoning, China, (2010).

[11] J. MacQueen, "Some methods for classification and analysis of multivariate observations", Proceedings of the fifth Berkeley symposium on mathematical statistics and probability, vol.1, no. 14, (1967), Berkeley, USA 1967, pp. 281-297.

[12] X. L. Qiu, “The Improvement and Application of K-means Algorithm”, Beijing University of Posts and Telecommunications, Beijing, China, (2012).

[13] J. Xie, S. Jiang, W. Xie and X. Gao, "An efficient global K-means clustering algorithm", Journal of computers, vol. 6, no. 2, (2011), pp. 271-279.

[14] D. Napoleon, P. G. Lakshmi, "An Enhanced k-means algorithm to improve the Efficiency Using Normal Distribution Data Points", International Journal on Computer Science and Engineering, vol. 2, no. 7, (2010), pp. 2409-2413.

[15] J. Serra, "Image analysis and mathematical morphology", Academic press, Salt Lake City, (1982).

[16] N. T. L. Anh, Y. C. Kim and G. S. Lee, "Morphological gradient applied to new active contour model for color image segmentation", Proceedings of the 6th International Conference on Ubiquitous Information Management and Communication, ACM, (2012) February 20-22, Kuala Lumpur, Malaysia.

[17] E. Aptoula and S. Lefèvre, "On lexicographical ordering in multivariate mathematical morphology", Pattern Recognition Letters, vol. 29, no. 2, (2008), pp. 109-118.

[18] J. Angulo, "Geometric algebra colour image representations and derived total orderings for morphological operators-Part I: Colour quaternions", Journal of Visual Communication and Image Representation, vol. 21, no. 1, (2010), pp. 33-48.

[19] M. K. Nayak, A. K. Talukdar and K. K. Sarma, "Hand Segmentation and Tracking of Continuous Hand Posture using Morphological Processing", International Journal of Electronics Signals and Systems (IJESS), vol. 3, no. 1, (2013), pp. 82-86. 
[20] S. M. Zabihi, M. Delgir and H. R. Pourreza, "Retinal vessel segmentation using color image morphology and local binary patterns", Proceedings of the 2010 6th IEEE Machine Vision and Image Processing (MVIP), (2010) October 27-28, Isfahan.

\section{Authors}

Zhang Qiu-yu, Researcher/PhD supervisor, graduated from Gansu University of Technology in 1986, and then worked at school of computer and communication in Lanzhou University of Technology. He is vice dean of Gansu manufacturing information engineering research center, a $\mathrm{CCF}$ senior member, a member of IEEE and ACM. His research interests include network and information security, information hiding and steganalysis analysis, image understanding and recognition, multimedia communication technology.

Lu Jun-chi, Graduated from Liaoning Technical University, Liaoning, China, in 2009. He is currently master student in Computer Applications, Lanzhou University of Technology, Lanzhou, China. His research interests include image processing and pattern recognition.

Zhang Mo-yi, PhD student, graduated from Lanzhou University of Technology in 2010, and then worked as the lecturer at the school of computer and communication in Lanzhou University of Technology. Her research interests include Image Processing and Pattern Recognition.

Duan Hong-xiang, PhD student, graduated from Xi'an University of Architecture and Technology with Bachelor of engineering degree in 1999, received master degree from Lanzhou University of Technology in 2011, and then worked at school of computer and communication in Lanzhou University of Technology. Her research interests include image processing and pattern recognition, dimensionality reduction for high-dimensional data.

Lv Lu, Graduate student in Computer Application Technology from Lanzhou University of Technology, Lanzhou, China. She obtained an undergraduate degree in Computer Science and Technology from Lanzhou University of Technology, Lanzhou, China, in 2012. Her research interests include image processing and pattern recognition, dynamic gesture trajectory recognition. 